\title{
Lichens of the Innvika Bay, Prins Oscars Land (Nordaustlandet, Svalbard)
}

\author{
Liudmila Konoreva $^{1 *}$, Sergey Chesnokov ${ }^{2}$ \\ ${ }^{1}$ Avrorin Polar-Alpine Botanical Garden-Institute of Kola Scientific Centre of RAS, \\ 184250 Kirovsk, Murmansk Region, Russia \\ ${ }^{2}$ Komarov Botanical Institute RAS, Professor Popov St. 2, 197376 St. Petersburg, Russia
}

\begin{abstract}
The paper presents a list of lichens for the Innvika Bay, which includes 157 lichenized and 2 lichenicolous fungi. Alectoria gowardii and Peltigera elisabethae are new to Svalbard. Furthermore, 36 other species were found new to Nordaustlandet. Twenty-seven species are rare on Svalbard, among them Aspilidea myrinii, Candelariella borealis, Gyalecta erythrozona, Miriquidica deusta, Rhizocarpon viridiatrum (reported for the second time), and Aspicilia cinerea, Cetraria nigricans, Cladonia arbuscula, Cystocoleus ebeneus, Lecidea ecrustacea, Peltigera frippii, Rhizocarpon eupetraeoides, Rinodina terrestris, Stereocaulon cumulatum, Toninia squalida, Verrucaria hydrela. Localities, substrates and distribution in Svalbard are given for each species. For rare and extremely rare species, all known locations are given. For some species, differences from closely related species are given.
\end{abstract}

Key words: Arctic, lichen biota, new records, Norway, rare species

DOI: $10.5817 / \mathrm{CPR} 2021-2-17$

\section{Introduction}

The Innvika Bay is part of the Prince Oscar Land located in the northern part of Nordaustlandet. Nordaustlandet is the second largest island in the Svalbard Archipelago. Its area is 14.443 square kilometres, three-quarters of which are covered by glaciers. The Prince Oscar Land is free from glaciers except for one little ice cap in the central part (Ahlmannfonna).
Climate of various parts of the island is quite different. The mean annual temperature is -7 to $-8^{\circ} \mathrm{C}$. The warmest months are July and August with mean daily temperature +2.3 to $+2.4^{\circ} \mathrm{C}$, the coldest - February or March $\left(-18\right.$ to $\left.-20^{\circ} \mathrm{C}\right)$.

According to the Norwegian bedrock map (Dallmann et al. 2002), the prevailing rocks on the northern part of the Prince

Received June 23, 2021, accepted December 20, 2021.

*Corresponding author: L. Konoreva <ajdarzapov@ynadex.ru>

Acknowledgements: The work of L. Konoreva was supported by the institutional research projects of the Avrorin Polar-Alpine Botanical Garden-Institute of Kola SC RAS entitled Plant and soil resources, improvement of management practices in protected natural areas in the context of climate change and anthropogenic impact and optimization of human habitat in Spitsbergen Archipelago, project No. AAAA-A17-117121120045-5. The work of S. Chesnokov was supported by the institutional research projects of the Komarov Botanical Institute of the Russian Academy of Sciences Flora of lichens and bryophytes of Russia and phytogeographically important regions, project No. AAAA-A19-119020690077-4. 
Oscar Land are Neoproterozoic quartzite and shale. The largest area (center and south-west) is formed by granite. According to geobotanical zoning of the Arctic, most of the Nordaustlandet, including the Prince Oscar Land, belongs to the Polar desert zone (Alexandrova 1977, Matveyeva et al. 2015).

There is fragmentary information about lichens of the Nordaustlandet (Fries 1867, Scholander 1934, Lynge 1938, Santesson 1939, Hagen 1950, Øvstedal et al. 2009, Nordin 2010, Zhurbenko and Brackel
2013 , etc.). The most complete list of lichens of the Nordaustlandet is presented on website 'Svalbard Lichen Database' [1], where 239 taxa are listed. Later, the authors of this paper added 58 species of lichens to the list (Konoreva et al. 2019, Czernyadjeva et al. 2020). Before our research, 293 species were known for Nordaustlandet, among them 186 species are widespread, 72 species are rare and 35 species are extremely rare. However, there is no information on lichens for Innvika Bay.

\section{Materials and Methods}

This work is a part of a comprehensive field study carried out in Svalbard by the Avrorin Polar-Alpine Botanical GardenInstitute Kola SC RAS. The lichens were collected by Liudmila Konoreva in the area of Innvika Bay (Nordaustlandet) in July 2011 (Fig. 1). About 500 lichen specimens were collected. Morphological and anatomical features of lichens were analyzed by standard light microscopy and chemical tests (Smith et al. 2009). Voucher lichen

specimens are deposited in the herbarium of the Avrorin Polar-Alpine Botanical Garden-Institute (KPABG). The geographical names and topographic bases for Fig. 1 respect the topographic map of Svalbard [2]. The nomenclature of lichens mainly follows Westberg et al. (2021) taking into account additions and changes published in a number of other studies (e.g. Lumbsch and Huhndorf 2010, Kondratyuk et al. 2020).

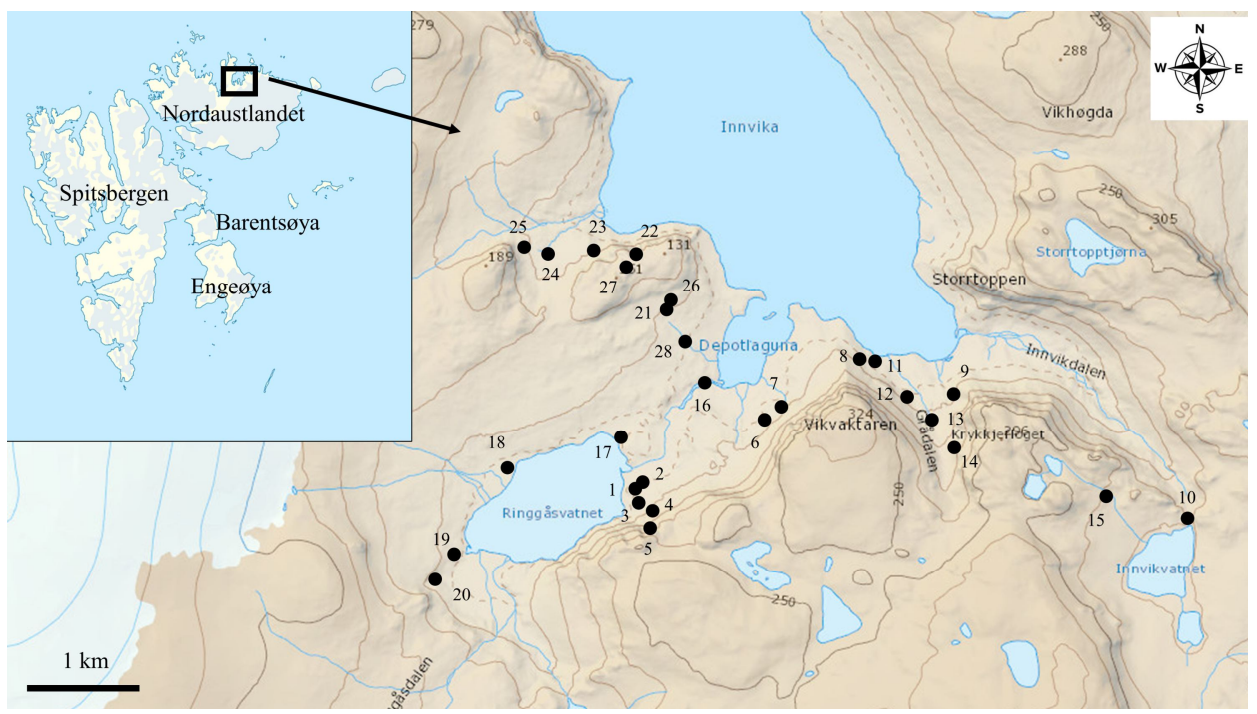

Fig. 1. Location of sampling sites in Innvika Bay. Source: 'TopoSvalbard'[2]. 


\section{List of localities:}

1. Nordaustlandet, Prince Oscars Land, Fotherby fjorden, south coast of Innvika Bay, SW coast of Depotlaguna, right bank of Ringgasdalen, $80^{\circ} 06^{\prime} 20.5^{\prime \prime} \mathrm{N}, 23^{\circ} 01^{\prime} 39.9^{\prime \prime} \mathrm{E}$, alt. $25 \mathrm{~m}$, stone rubble, 23.07.2011

2. ibidem, $80^{\circ} 06^{\prime} 18.1^{\prime \prime} \mathrm{N}, 23^{\circ} 01^{\prime} 31.3^{\prime \prime} \mathrm{E}$, alt. $27 \mathrm{~m}$, rocky tundra, 23.07.2011

3. ibidem, $80^{\circ} 06^{\prime} 15.4^{\prime \prime} \mathrm{N}, 23^{\circ} 01^{\prime} 31.2^{\prime \prime} \mathrm{E}$, alt. $32 \mathrm{~m}$, rocky tundra near the lake, 23.07.2011

4. ibidem, $80^{\circ} 06^{\prime} 12.9^{\prime \prime} \mathrm{N}, 23^{\circ} 01^{\prime} 46.5^{\prime \prime} \mathrm{E}$, alt. $74 \mathrm{~m}$, rocks, 23.07 .2011

5. ibidem, $80^{\circ} 06^{\prime} 08.8^{\prime \prime} \mathrm{N}, 23^{\circ} 01^{\prime} 41.8^{\prime \prime} \mathrm{E}$, alt. $94 \mathrm{~m}$, seashore colony of birds, 23.07 .2011

6. ibidem, west slope of Vikvaktaren, $80^{\circ} 06^{\prime} 33^{\prime \prime} \mathrm{N}, 23^{\circ} 05^{\prime} 13^{\prime \prime} \mathrm{E}$, alt. $35 \mathrm{~m}$, huge boulders with numerous rivuletes, 24.07.2011

7. ibidem, foot of west slope Vikvaktaren, $80^{\circ} 06^{\prime} 36^{\prime \prime} \mathrm{N}, 23^{\circ} 05^{\prime} 39^{\prime \prime} \mathrm{E}$, alt. $38 \mathrm{~m}$, bryophyte community under snow field, 24.07.2011

8. ibidem, $80^{\circ} 06^{\prime} 46.4^{\prime \prime} \mathrm{N}, 23^{\circ} 08^{\prime} 26.9^{\prime \prime} \mathrm{E}$, alt. $16 \mathrm{~m}$, slope by the stream on the bay, 25.07.2011

9. ibidem, Grådalen, foot of the slope Krykkjefloget, $80^{\circ} 06^{\prime} 32.0^{\prime \prime} \mathrm{N}, 23^{\circ} 10^{\prime} 31.1^{\prime \prime} \mathrm{E}$, alt. $45 \mathrm{~m}$, near seashore colony of birds, 25.07.2011

10. ibidem, near Innvikvatnet, $80^{\circ} 05^{\prime} 48.7^{\prime \prime} \mathrm{N}, 23^{\circ} 16^{\prime} 32.9^{\prime \prime} \mathrm{E}$, alt. $157 \mathrm{~m}$, rocks on the bank of lake, 25.07.2011

11. ibidem, steep north-northeast slope of Vikvaktaren, $80^{\circ} 06^{\prime} 47^{\prime \prime} \mathrm{N}, 23^{\circ} 07^{\prime} 59^{\prime \prime} \mathrm{E}$, alt. 45 $\mathrm{m}$, with huge boulders covered by vegetation, 26.07.2011

12. ibidem, Grådalen, $80^{\circ} 06^{\prime} 33^{\prime \prime} \mathrm{N}, 23^{\circ} 09^{\prime} 25^{\prime \prime} \mathrm{E}$, alt. $28 \mathrm{~m}$, left bank of the river near of seashore colony of birds, 26.07.2011

13. ibidem, $80^{\circ} 06^{\prime} 26^{\prime \prime} \mathrm{N}, 23^{\circ} 09^{\prime} 42^{\prime \prime} \mathrm{E}$, alt. $57 \mathrm{~m}$, rock field on left bank of river, 26.07.2011

14. ibidem, $80^{\circ} 06^{\prime} 18^{\prime \prime} \mathrm{N}, 23^{\circ} 10^{\prime} 18^{\prime \prime} \mathrm{E}$, alt. $141 \mathrm{~m}$, right bank of river rock outcrops on western slope of Krykkjefloget, 26.07.2011

15. ibidem, near Innvikvatnet, $80^{\circ} 05^{\prime} 56^{\prime \prime} \mathrm{N}, 23^{\circ} 14^{\prime} 20^{\prime \prime} \mathrm{E}$, alt. $184 \mathrm{~m}$, tundra, 26.07 .2011

16. ibidem, SW coast of Depotlaguna, right bank of Ringasdalen, $80^{\circ} 06^{\prime} 45^{\prime \prime} \mathrm{N}$, $23^{\circ} 03^{\prime} 52^{\prime \prime} \mathrm{E}$, alt. $4 \mathrm{~m}$, gentle slope to the river, 27.07.2011

17. ibidem, Ringgåsdalen, north-east end of Ringgåsvatnet, $80^{\circ} 06^{\prime} 35^{\prime \prime} \mathrm{N}, 23^{\circ} 01^{\prime} 20^{\prime \prime} \mathrm{E}$, alt. $19 \mathrm{~m}$, stone rubble, 28.07.2011

18. ibidem, north-west bank of Ringgåsvatnet, $80^{\circ} 06^{\prime} 30^{\prime \prime} \mathrm{N}, 22^{\circ} 58^{\prime} 00^{\prime \prime} \mathrm{E}$, alt. $12 \mathrm{~m}$, left bank of steam, 28.07.2011

19. ibidem, west bank of Ringgåsvatnet, $80^{\circ} 06^{\prime} 10^{\prime \prime} \mathrm{N}, 22^{\circ} 56^{\prime} 18^{\prime \prime} \mathrm{E}$, alt. $40 \mathrm{~m}$, glacial terrace on lake shore, 28.07.2011

20. ibidem, Ringgåsdalen, $80^{\circ} 06^{\prime} 02.5^{\prime \prime} \mathrm{N}, 22^{\circ} 55^{\prime} 44.6^{\prime \prime} \mathrm{E}$, alt. $34 \mathrm{~m}$, rocks on the southwestern part of Ringgåsvatnet, 28.07.2011

21. ibidem, west coast of Innvika Bay, north-west coast of Depotlaguna, left bank of Ringasdalen River, $80^{\circ} 07^{\prime} 09.4^{\prime \prime} \mathrm{N}, 23^{\circ} 02^{\prime} 59.8^{\prime \prime} \mathrm{E}$, alt. $44 \mathrm{~m}$, moss-lichen-cereal tundra with stone placers and flowing streams, 29.07-30.07.2011

22. ibidem, $80^{\circ} 07^{\prime} 23.6^{\prime \prime} \mathrm{N}, 23^{\circ} 02^{\prime} 25.4^{\prime \prime} \mathrm{E}$, alt. $115 \mathrm{~m}$, rocks, 29.07 .2011

23. ibidem, $80^{\circ} 07^{\prime} 28.3^{\prime \prime} \mathrm{N}, 23^{\circ} 01^{\prime} 20.1^{\prime \prime} \mathrm{E}$, alt. $49 \mathrm{~m}$, stone rubble, 29.07.2011

24. ibidem, $80^{\circ} 07^{\prime} 29.9^{\prime \prime} \mathrm{N}, 23^{\circ} 00^{\prime} 12.1^{\prime \prime} \mathrm{E}$, alt. $41 \mathrm{~m}$, river floodplain, 29.07.2011

25. ibidem, $80^{\circ} 07^{\prime} 31.8^{\prime \prime} \mathrm{N}, 22^{\circ} 59^{\prime} 23.5^{\prime \prime} \mathrm{E}$, alt. $45 \mathrm{~m}$, shale rocks, 29.07 .2011

26. ibidem, $80^{\circ} 07^{\prime} 10^{\prime \prime} \mathrm{N}, 23^{\circ} 03^{\prime} 01^{\prime \prime} \mathrm{E}$, alt. $56 \mathrm{~m}$, south facing slope with rivulets and boulders, 30.07.2011 
27. ibidem, $80^{\circ} 07^{\prime} 23^{\prime \prime} \mathrm{N}, 23^{\circ} 02^{\prime} 16^{\prime \prime} \mathrm{E}$, alt. $104 \mathrm{~m}$, mountain terrace with snow fields, huge boulders, 30.07.2011

28. ibidem, $80^{\circ} 07^{\prime} 00^{\prime \prime} \mathrm{N}, 23^{\circ} 03^{\prime} 23^{\prime \prime} \mathrm{E}$, alt. $29 \mathrm{~m}$, valley of rocky stream on terrace, 30.07.2011

\section{Results}

The study resulted in an annotated list of species, which includes 157 lichenized and 2 lichenicolous fungi. Localities, substrates and distribution in Svalbard are given for each species. The distribution of the species in Svalbard was estimated according to the studies of Elvebakk and Hertel (1996), Osyczka (2006), Krzewicka and Maciejowski (2008), Urbanavichene and Koroleva (2008), Øvstedal et al. (2009), Ziaja et al. (2009), Redchenko et al. (2010), Konoreva (2011), Singh and Ravindra (2013), Zhurbenko and Brackel (2013), Węgrzyn et al. (2015), Breuss (2017), Wietrzyk et al. (2017), Konoreva and Chesnokov (2018), Maciejowski et al. (2018), Inoue et al. (2019), Konoreva et al. (2019), and Svalbard Lichens Database [1]. For rare and extremely rare species, all known locations on the Svalbard are given. For some species, differences from closely related species are given.

All identified species are new to Innvika Bay, among them Alectoria gowardii and Peltigera elisabethae are new to Svalbard, 36 species are new to Nordaustlandet. Twenty-seven species are rare and extremely rare on Svalbard, among them Aspilidea myrinii, Candelariella borealis, Gyalecta erythrozona, Miriquidica deusta, Rhizocarpon viridiatrum reported for the second time, and Aspicilia cinerea, Cetraria nigricans, Cladonia arbuscula, Cystocoleus ebeneus, Lecidea ecrustacea, Peltigera frippii, Rhizocarpon eupetraeoides, Rinodina terrestris, Stereocaulon cumulatum, Toninia squalida, Verrucaria hydrela - for the third time for Svalbard.

The following abbreviations and notation are in the list:

$!$ - new to Svalbard;

* - new to Nordaustlandet;

+- lichenicolous fungi;

KPABG - herbarium of the Polar-Alpine Botanical Garden-Institute, Kirovsk,

Murmansk Region, Russia;

$\mathrm{O}$ - herbarium of the University of Oslo;

BG - herbarium of the University of Bergen;

TROM - herbarium of the Arctic University of Norway, Tromsø.

\section{The list of species}

Acarospora veronensis A. Massal. - 26 (KPABG 13485), on siliceous stone. Widely distributed throughout Svalbard.

*Adelolecia kolaënsis (Nyl.) Hertel \& Rambold - 4, 16, 20 (KPABG 13631), on siliceous stones. Widely distributed throughout Svalbard, but easily overlooked species.

*Agonimia gelatinosa (Ach.) M. Brand \& Diederich - 16, on mosses. Widely distributed throughout Svalbard. 
:Alectoria gowardii Lumbsch - 1 (KPABG 13640), on soil. The species was described by Halonen et al. (2009) as Gowardia arctica Halonen et al. It is distinguished from the closely related Alectoria nigricans by shiny thallus and mainly uniform color, as well as the absence of well-defined main branches. Alectoria gowardii could be confused with Bryoria nitidula (Th. Fr.) Brodo \& D. Hawksw. and Bryocaulon divergens (Ach.) Kärnefelt. However, Bryoria nitidula has dark-colored pseudocyphellae and produces fumarprotocetraric acid (K-, PD+ red); and Bryocaulon divergens is readily separated by its red-brown color. In the world, the species is known from northern regions of Canada and Russia along the Arctic Ocean coast and islands (Halonen et al. 2009).

Alectoria nigricans (Ach.) Nyl. - 4 (KPABG 13610, 13622), 6 (KPABG 13575), 11, 17 , 25 (KPABG 13493), 26 (KPABG 13535), on soil, on soil over the boulders, on deadwood. Widely distributed throughout Svalbard and common.

Alectoria ochroleuca (Hoffm.) A. Massal. - 4 (KPABG 13519, 13623), 20 (KPABG 13633), on soil, among the stones, on rocks. Widely distributed throughout Svalbard.

Allantoparmelia alpicola (Th. Fr.) Essl. - 1, 4, 13 (KPABG 13643), 15 (KPABG 13585), 17 (KPABG 13502), on siliceous stones. Widely distributed throughout Svalbard.

*Amandinea coniops (Wahlenb.) M.Choisy ex Scheid. \& H.Mayrhofer - 21, on siliceous stone. Widely distributed throughout Svalbard, especially along the seashore colony of birds and rocks.

Arthrorhaphis citrinella (Ach.) Poelt - 4 (KPABG 13610), 9, 12 (KPABG 13628), on soil. Widely distributed throughout Svalbard.

* Aspicilia cinerea (L.) Körb. - 16 (KPABG 13564), on stone. Rare on Svalbard. Reported to the third time from Svalbard. Previously reported from Sørkapp Land (Øvstedal et al. 2009) and Nordenskiöld land - between Vestpynten and Bjørndalen (O-L116816) [1].

*Aspilidea myrinii (Fr.) Hafellner - 16 (KPABG), on stone. Rare on Svalbard. Reported to the second time from Svalbard. Previously reported from Davishamna (Øvstedal et al. 2009).

*Baeomyces carneus (Retz.) Flörke - 8 (KPABG), on soil. Rare on Svalbard. Known from three localities of Nordenskiöld Land - Reindalen, Grøndalen and Adventdalen (Urbanavichene and Koroleva 2008, Øvstedal et al. 2009).

Biatora cuprea (Sommerf.) Fr. - 4 (KPABG 13512), on soil. Widely distributed throughout Svalbard, but not common.

Biatora ementiens (Nyl.) Printzen - 9 (KPABG), on mosses and soil. Widely distributed throughout Svalbard, but not common.

Biatora subduplex (Nyl.) Rasanen ex Printzen - 11 (KPABG 13616), 16 (KPABG 13556, 13595), 21, on soil, on plant debris, on mosses. Widely distributed throughout Svalbard and common.

Blastenia ammiospila (Wahlenb.) Arup et al. - 20, on mosses. Widely distributed throughout Svalbard and common.

Brodoa oroarctica (Krog) Goward - 4 (KPABG 13621), on siliceous stones. Widely distributed throughout Svalbard.

Bryocaulon divergens (Ach.) Karnefelt - 26 (KPABG 13536), on soil. Widely distributed throughout Svalbard and common.

*Bryonora castanea (Hepp) Poelt - 16, on soil and mosses. Sporadically distributed throughout Svalbard. 
Bryonora septentrionalis Holt.-Hartw. - 16, on soil and mosses. Widely distributed throughout Svalbard.

*Bryoplaca jungermanniae (Vahl) Søchting et al. - 5, on mosses. Widely distributed throughout Svalbard.

Bryoplaca tetraspora (Nyl.) Søchting et al. - 1 (KPABG 13480, 13614), on soil. Widely distributed throughout Svalbard.

Calvitimela armeniaca (DC.) Hafellner - 6, 9, 15, on siliceous stones. Widely distributed throughout Svalbard.

Candelariella aurella (Hoffm.) Zahlbr. - 16, on limestone. Widely distributed throughout Svalbard.

Candelariella borealis M. Westb. - 21, on soil and mosses. Rare on Svalbard. Reported to the second time from Svalbard. Previously reported from Nordaustlandet Damflya (Konoreva et al. 2019). Candelariella borealis very similar to C. placodizans, but distinguish by larger and darker yellow thallus with a smooth surface and squamules uneven with swollen tips (Westberg 2007).

*Candelariella placodizans (Nyl.) H. Magn. - 9 (KPABG 13538), 25 (KPABG), on soil. Sporadically distributed throughout Svalbard. Candelariella placodizans characterized by greenish yellow to pale yellow thallus and squamules granular to flattened, with pulverulent surface (Westberg 2007).

Candelariella vitellina (Hoffm.) Mull. Arg. - 9 (KPABG 13509), 16, 26 (KPABG 13545), on siliceous stones. Widely distributed throughout Svalbard and common.

Cetraria aculeata (Schreb.) Fr. - 16 (KPABG 13557), on soil. Widely distributed throughout Svalbard and common.

Cetraria ericetorum Opiz - 26 (KPABG 13532), on soil. Widely distributed throughout Svalbard.

Cetraria islandica (L.) Ach. - 6 (KPABG 13626), 7 (KPABG 13548), 10 (KPABG 13543), 21, 23 (KPABG 13499), on soil among mosses. Widely distributed throughout Svalbard.

Cetraria muricata (Ach.) Eckfeldt - 1, 16 (KPABG 13593), on soil. Widely distributed throughout Svalbard, although it is far less common than C. aculeata.

Cetraria nigricans Nyl. - 1 (KPABG 13638), on soil. Rare on Svalbard. Reported to the third time from Svalbard. Previously reported from two localities from Nordaustlandet - Duvepynten (Øvstedal et al. 2009) and Damflya (Konoreva et al. 2019).

Cetrariella delisei (Bory ex Schaer.) Karnefelt \& Thell - 1 (KPABG 13470), 8 (KPABG 13606) 25 (KPABG 13491), 25, on soil among mosses in swampy communities. Widely distributed throughout Svalbard.

Cladonia amaurocraea (Florke) Schaer. - 21 (KPABG 13555), on soil among mosses. Widely distributed throughout Svalbard.

*Cladonia arbuscula (Wallr.) Flot. - 17 (KPABG 13601), on soil among mosses. Rare on Svalbard. Reported to the third time from Svalbard. Previously reported from two localities from Edgeøya - Kvalpynten (Ahti 1961) and Liefdefjorden (Øvstedal et al. 2009).

Cladonia coccifera (L.) Willd. - 1 (KPABG 13476, 13483), 4, 8 (KPABG 13550), 11 (KPABG 13615), 25, 26 (KPABG 13536), on soil among stones, on mosses, on driftwood. Widely distributed throughout Svalbard.

*Cladonia deformis (L.) Hoffm. - 25, 26 (KPABG 13531), on soil and mosses. Widely distributed throughout Svalbard, but not common.

Cladonia gracilis ssp. elongata (Wulfen) Vain. - 1 (KPABG 13475), 4 (KPABG 13525), 5 (KPABG 13515), 6 (KPABG 13574), 8 (KPABG 13550), 17, 23 (KPABG 
13498), 25 (KPABG 13490), on soil among mosses. Widely distributed throughout Svalbard and very common.

Cladonia mitis Sandst. - 2, 4 (KPABG 13516), 17, on soil among mosses. Widespread along the whole coast of Svalbard.

Cladonia pleurota (Florke) Schaer. - 4 (KPABG 13609), 6, 25 (KPABG 13492), on soil. Widely distributed throughout Svalbard.

Cladonia pyxidata (L.) Hoffm. - 4 (KPABG 13612), 10 (KPABG 13543), 16, 25, on soil. Widely distributed throughout Svalbard and common.

Cladonia stricta (Nyl.) Nyl. - 25, on soil. Sporadically distributed throughout Svalbard.

Cystocoleus ebeneus (Dillwyn) Thwaites - 2, on soil. Rare on Svalbard. Reported to the third time from Svalbard. Previously reported from Nordaustlandet - Wargentindalen; Haakon VII Land - Bockfjorden (Øvstedal et al. 2009).

Dactylina ramulosa (Hook.) Tuck. - 9 (KPABG 13539), 16 (KPABG 13590, 13594), on soil. Widely distributed in the north and north-west parts of Svalbard and is rare in the southern regions.

+Endococcus propinquus (Körb.) Trevis. s. lat. - 1, on thallus of Rhizocarpon sp. Rare on Svalbard. These reports refer to Endococcus propinquus s. lat., becaus E. propinquus s. str. is restricted to hosts of the genus Porpidia (Sérusiaux et al. 1999). Endococcus propinquus s. lat. known from a few scattered sites of Svalbard: Sørkapp Land - Hornsund; Albert I Land - Kobbefjorden; Ny-Friesland - Sorgfjorden; Nordaustlandet - Wahlenbergfjorden; Bjørnøya (Zhurbenko and Brackel 2013). Endococcus propinquus s. str. known only Wedel Jarlsberg Land - Rålstranda and Låkpynten (Kukwa and Jabłońska 2008).

*Farnoldia micropsis (A. Massal.) Hertel - 14, on limestone. Known from a few scattered sites of Svalbard: Hinlopenstretet (Fries 1867); Bjørnøya (Lynge 1926); Amsterdamøya (Hertet and Ullrich 1976); Oscar II Land - Ny-Ålesund area (Hertel 1977), Beach from Brandalpynten to Stuphallet, Bayelva (Breuss 2017); Axeløya; Dickson Land - Lyckholmdalen (Øvstedal et al. 2009).

Flavocetraria cucullata (Bellardi) Karnefelt \& Thell - 8 (KPABG 13605), 25 (KPABG 13489), on soil. Widely distributed throughout Svalbard.

Flavocetraria nivalis (L.) Karnefelt \& Thell - 1 (KPABG 13637), 4 (KPABG 13623), 14, 15 (KPABG 13584), 20 (KPABG 13633), on soil. Widely distributed throughout Svalbard.

*Gyalecta erythrozona Lettau - 8, on stone. Rare on Svalbard. Reported to the second time from Svalbard. Previously reported from Nordenskiöld Land - Bjørndalen (Øvstedal et al. 2009).

Gyalolechia bracteata (Hoffm.) A. Massal. - 16, on soil. Widely distributed throughout Svalbard.

Henrica melaspora (Taylor) S. Savić \& Tibell - 16 (KPABG 13591), on siliceous stones. Rare on Svalbard. Known from a few scattered sites of Svalbard: Bjørnøya (Lynge 1926), Brøggerhalvøya - Ny-Ålesund area (Hertel 1977), Gluudneset (Øvstedal et al. 2009); Nordenskiöld Land - Kapp Linné (Øvstedal et al. 2009); Nordaustlandet - Damflya (Konoreva et al. 2019).

Hypogymnia austerodes (Nyl.) Räsänen - 4 (KPABG 13512), on siliceous stone. Rare on Svalbard. By Øvstedal et al. (2009) Hypogymnia subobscura (Vain.) Poelt included in H. austerodes. According Westberg et al. (2011) H. subobscura is a separate taxon and differs from $H$. austerodes in having papillae that never breaks up into soralia and in lacking 3-hydroxyphysodic acid. Known from a few scattered sites 
of Svalbard (according Elvabakk and Hertel 1996): Haakon VII Land - Siktefjellet, Bünsow Land - Gipsdalen.

Ionaspis lacustris (With.) Lutzoni - 24, on wet siliceous stone. Widely distributed throughout Svalbard.

Japewia tornoensis (Nyl.) Tønsberg - 14, 28 (KPABG 13484, 13505), on soil and mosses, on driftwood. Widely distributed throughout Svalbard.

*+Lasiosphaeriopsis stereocaulicola (Th. Fr. ex Linds.) O. E. Erikss. \& R. Sant. - 14, on phyllocladia of Stereocaulon sp. Widely distributed throughout Svalbard.

Lecanora epibryon (Ach.) Ach. - 7 (KPABG 13546), on soil and mosses. Widely distributed throughout Svalbard.

Lecanora intricata (Ach.) Ach. - 4 (KPABG 13525), 19 (KPABG 13603), 23, on siliceous stones. Known from a few scattered sites of Svalbard: Sørkapp Land and Sørkappøya (Lynge 1924); Edgeøya (Aptroot and Aistrup 1991); Barentsøya Steinfjorden; Nordenskiöld Land - Bjørndalen (Øvstedal et al. 2009), Nordenskiöldfjellet (Breuss 2017), coast from Colesbay to Grumant (Konoreva and Chesnokov 2018); Danskøya - Virgohamna (Øvstedal et al. 2009); Nordaustlandet Damflya (Konoreva et al. 2019).

Lecanora polytropa (Ehrh. ex Hoffm.) Rabenh. - 1, 4 (KPABG 13619), 9, 11, 16 (KPABG 13558), 20 (KPABG 13631), on siliceous stones. Widely distributed throughout Svalbard.

Lecidea atrobrunnea (Ramond ex Lam. \& DC.) Schaer. - 9, on siliceous stones. Widely distributed throughout Svalbard.

Lecidea auriculata Th. Fr. $-\mathbf{8}, \mathbf{1 5}, \mathbf{1 7}$, on siliceous stones. Widely distributed throughout Svalbard.

*Lecidea confluens (Weber) Ach. - 16, 19, 21, on siliceous stones. Sporadically distributed throughout Svalbard.

* Lecidea ecrustacea (Anzi ex Arnold) Arnold - 4, on siliceous stone. Rare on Svalbard. Reported to the third time from Svalbard. Previously reported from Nordenskiöld Land - Camp Morton (Hertel 1970), Longyearbyen area (Hartmann 1980).

Lecidea lapicida (Ach.) Ach. - 20, on siliceous stone. Widely distributed throughout Svalbard.

Lecidea lapicida var. pantherina Ach. - 16, 21, on siliceous stones. Widely distributed throughout Svalbard.

Lecidea ramulosa Th. Fr. - 16, on soil. Widely distributed throughout Svalbard.

Lecidea swartzioidea Nyl. - 17 (KPABG 13504), on siliceous stone. Rare on Svalbard. Known from a few scattered sites of Svalbard: Amsterdamøya (Hertel and Ullrich 1976); Nordenskiöld Land - Longyearbyen (as L. cf. swartzoidea; Hartmann 1980); Wedel Jarlsberg Land - Mariaholmen (Øvstedal et al. 2009); Oskar II Land Zeppelinfjellet (Breuss 2017); Nordaustlandet - Damflya (Konoreva et al. 2019).

Lecidoma demissum (Rutstr.) Gotth. Schneid. \& Hertel - 1, on soil. Widely distributed throughout Svalbard.

*Lendemeriella exsecuta (Nyl.) S. Y. Kondr. - 21, on siliceous stone. Rare on Svalbard. Known from a few scattered sites of Svalbard: Nordenskiöld Land - Longyeardalen, Reindalen; Oskar II Land - Thusbukta at Ny-Ålesund (Søchting et al. 2008), Kaffiøyra (Węgrzyn et al. 2015); Bjørnøya as Blastenia arctica (Lynge 1926); Haakon VII Land - Möllerfjorden (Søchting 1989).

Lendemeriella tornoensis (H. Magn.) S.Y. Kondr. - 16, on mosses. Rare on Svalbard. Known from a few scattered sites of Svalbard: Sabine Land - Sassendalen (Søchting et al. 2008); Nordaustlandet - Damflya (Konoreva et al. 2019); Nordenskiöld Land - 
Reindalen (Søchting 1992); Funglesongen Nordvestøyane - Ytre Norskøya (O-L160168) [1].

Lepraria gelida Tønsberg \& Zhurb. - 9 (KPABG 13542), 16, on soil and mosses. Spot tests: $\mathrm{K}+$ yellow to orange, $\mathrm{C}-, \mathrm{KC}+\mathrm{red}, \mathrm{Pd}+$ yellow to orange; contains alectorialic and porphyrilic acids (Øvstedal et al. 2009, Saag et al. 2009). Lepraria gelida can be confused with Lepraria eburnea J. R. Laundon, but is distinct by a granular or wartygranular thallus with granular soredia (L. eburnea has powdery to cottony thallus with farinose soredia) and present porphyrilic acid (Saag et al. 2009, Makarova and Himelbrant 2008). Widely distributed throughout Svalbard.

Lepraria neglecta (Nyl.) Lettau - 1, 4, 8, 10, 12, 14, 21, on soil and mosses, on stones. Spot tests: $\mathrm{K}+$ yellow, $\mathrm{C}+$ reddish orange, $\mathrm{KC}+$ reddish orange, $\mathrm{Pd}+$ lemon yellow; contains alectorialic acid and angardianic/roccellic acid (Øvstedal et al. 2009, Saag et al. 2009). Lepraria neglecta may resemble L. gelida, but is distinct by its bluish colour, the presence of granular soredia, absence of a medulla, and presence of a fatty acid (Øvstedal et al. 2009, Saag et al. 2009). Widely distributed throughout Svalbard.

*Lichenomphalia umbellifera (L.:Fr.) Redhead et al. - 6 (KPABG 13602), 9 (KPABG 13537), on soil and mosses. Widely distributed throughout Svalbard.

Lopadium coralloideum (Nyl.) Lynge - 1, 8, 16 (KPABG 13592), 23 (KPABG 13496), 25 (KPABG 13495), on mosses and soil. Widely distributed throughout Svalbard.

Lopadium pezizoideum (Ach.) Körb. - 11, 21, 25, on mosses and soil. Widely distributed throughout Svalbard.

Melanelia hepatizon (Ach.) A.Thell - 4 (KPABG 13523), 6 (KPABG 13571), on siliceous stones. Widely distributed throughout Svalbard.

Melanelia stygia (L.) Essl. - 1, 4 (KPABG 13619), 6 (KPABG 13568), 15, 27 (KPABG 13528), on siliceous stones. Widely distributed throughout Svalbard.

Melanohalea infumata (Nyl.) O. Blanco et al. - 18 (KPABG 13540), on siliceous stone. Widely distributed throughout Svalbard.

Micarea incrassata Hedl. - 20, on soil. Known from a few scattered sites of Svalbard: Bjørnøya (Lynge 1926); Oskar II Land - Ny-Ålesund (Hertel 1977), Saddle between Zeppelinfjellet and Lundryggen (Breuss 2017), Austre Brøggerbreen (Inoue et al. 2019); Nordenskiöld Land - Kapp Laila (Øvstedal et al. 2009), Rieperbreen (Wietrzyk et al. 2017); Ny-Friesland - between Austbotnhytta and Smutsdalen (Øvstedal et al. 2009); Dickson Land - Petuniabukta (Redchenko et al. 2010); Noraustlandet - Damflya (Konoreva et al. 2019).

* Miriquidica deusta (Stenh.) Hertel \& Rambold - 16, on siliceous stone. Rare on Svalbard. Reported to the second time from Svalbard. Previously reported from Colesdalen (Øvstedal et al. 2009).

Miriquidica lulensis (Hellb.) Hertel \& Rambold - 16, on siliceous stone. Widely distributed throughout Svalbard.

Miriquidica nigroleprosa (Vain.) Hertel \& Rambold - 4 (KPABG 13618), 6, 10, on siliceous stones. Known from a few scattered sites of Svalbard: Blomstrandhalvøya (Hertel 1977); Amsterdamøya (Hertel and Ullrich 1976, Søchting et al. 2008); Haakon VII Land - Bockfjorden (Hafellner 1982); Sørkapp Land (Olech 1990); Nordaustlandet - Phippsøia, Lågøya (Øvstedal et al. 2009), Damflya (Konoreva et al. 2019).

*Myriospora smaragdula (Wahlenb. ex Ach.) Nägeli ex Uloth - 21 (KPABG 13458), on siliceous stone. Widely distributed throughout Svalbard.

Ochrolechia frigida (Sw.) Lynge - 1 (KPABG 13637), 4 (KPABG 13519, 13525), 6 (KPABG 13626), 8 (KPABG 13526), 9 (KPABG 13538), 14, 15 (KPABG 13585), 
16, 21, 23 (KPABG 13496, 13634), 25, 26 (KPABG 13545), on mosses soil and stone. Widely distributed throughout Svalbard.

Orphniospora moriopsis (A. Massal.) D. Hawksw. - 15, 16, on siliceous stones. Widely distributed throughout Svalbard.

Parmelia omphalodes (L.) Ach. - 6 (KPABG 13576), 8 (KPABG 13604), 22, 23, 25, on soil over siliceous stones and mosses. Widely distributed throughout Svalbard.

Parmelia saxatilis (L.) Ach. - 6, on siliceous stone. Widely distributed throughout Svalbard.

Parmelia skultii Hale - 4 (KPABG 13524), 6 (KPABG 13580), 14, on soil. Widely distributed throughout Svalbard.

Peltigera aphthosa (L.) Willd. - 5 (KPABG 13586), on soil and mosses. Widely distributed throughout Svalbard.

Peltigera canina (L.) Willd. - 1, 5 (KPABG 13587), 6 (KPABG 13625), 8 (KPABG 13607), 18, on soil and mosses. Widely distributed throughout Svalbard.

Peltigera didactyla (With.) J. R. Laundon - 9, 21 (KPABG 13461), on soil. Widely distributed throughout Svalbard.

!Peltigera elisabethae Gyeln. - 6, on soil and mosses. Probably much overlooked, but usually easily distinguished by schizidia-like propagules, veinless lower surface but with small pale or white interstices (Vitikainen 2007). In the arctic, the species is known from Europe, Asia, North America (Vitikainen 2007).

Peltigera frippii Holt.-Hartw. - 1 (KPABG 13476), among mosses. Rare on Svalbard. Reported to the third time from Svalbard. Previously reported only from Nordaustlandet - Lady Franklinfjorden and Brennevinsfjorden (Vitikainen 1994).

Peltigera kristinssonii Vitik. - 6, on soil and mosses. Rare on Svalbard. Known from a few scattered sites of Svalbard: Bjørnøya (Engelskjøn 1986); Nordaustlandet Murchisonfjorden; Nordenskiöld Land - Linnedalen; Oskar II Land - Gluudneset (Elvebakk and Hertel 1996).

Peltigera leucophlebia (Nyl.) Gyeln. - 1, 6 (KPABG 13624), 21 (KPABG 13554), on soil and among mosses. Widely distributed throughout Svalbard.

Peltigera lyngei Gyeln. - 1 (KPABG 13477), 4 (KPABG 13520), 7 (KPABG 13547), 18 (KPABG 13486), 27 (KPABG 13534), on soil, among mosses and driftwood. Rare on Svalbard. Known from a few scattered sites of Svalbard: Danskøya - Kobbefjorden (Gyelnik 1932); Nordenskiöld Land - Sveagruva (as P. malacea var. lyngei; Eurola 1971), Grønfjorden, Adventfjorden; Nordaustlandet - Nordkapp, Floraberget (Vitikainen 1994).

Peltigera malacea (Ach.) Funck - 6 (KPABG 13570), on soil. Widely distributed throughout Svalbard.

Peltigera rufescens (Weiss) Humb. - 5 (KPABG 13588), 7 (KPABG 13549), 16 (KPABG 13596), 21 (KPABG 13462, 13553), 25 (KPABG 13635), on soil among mosses, in depressions. Widely distributed throughout Svalbard.

Peltigera scabrosa Th. Fr. - 6, on soil. Widely distributed throughout Svalbard.

Peltigera venosa (L.) Hoffm. - 5 (KPABG 13589), 16 (KPABG 13562), on soil in wet conditions. Widely distributed throughout Svalbard.

Pertusaria geminipara (Th. Fr.) C.Knight ex Brodo - 25, on soil. Widely distributed throughout Svalbard.

Pertusaria oculata (Dicks.) Th. Fr. - 1 (KPABG 13481), 4 (KPABG 13517, 13518, 13525, 13611), 9 (KPABG 13538), 10 (KPABG 13543), 16 (KPABG 13597), 21, 25 (KPABG 13488, 13494), 26 (KPABG 13533), on soil among stones, over mosses. Widely distributed throughout Svalbard. 
Physcia caesia (Hoffm.) Furnr. - 23 (KPABG 13497), on siliceous stone. Widely distributed throughout Svalbard and common.

Pleopsidium chlorophanum (Wahlenb.) Zopf - 4 (KPABG 13598), 9 (KPABG 13600), 15 (KPABG 13599), on siliceous stones. Widely distributed throughout Svalbard and common.

*Polyblastia sendtneri Kremp. - 16, on soil. Widely distributed throughout Svalbard and common.

Polyblastia septentrionalis Lynge - 16, on siliceous stone. Rare on Svalbard. Known from a few scattered sites of Svalbard: Nordaustlandet - Brennevinsfjorden; N end of Prins Karls Forland (Øvstedal et al. 2009); Nordenskiöld Land - Nordenskiöldfjellet (Breuss 2017); Nathorst Land - Cape Blix (O-L168356) [1].

*Polysporina simplex (Borrer ex Hook.) Vězda - 16, on siliceous stone. Known from a few scattered sites of Svalbard: Kong Karls Land - Abeløya (Lynge 1939), Hårfagrehaugen (Øvstedal et al. 2009); Hornsund (Nowak 1965); Sørkapp Land (Olech 1990); Bünsow Land - Gipsdalen (Elvebakk and Hertel 1996); Nathorst Land - Forsbladhamna (O-L156328), Midterhuken (O-L156327), Kap Blix (O-L156326); Akseløya (O-L156325); Nordenskiöld Land - Between Vestpynten and Bjørndalen (O-L116687) [1], Rieperbreen (Wietrzyk et al. 2017); Oskar II Land - Irenebreen (Wietrzyk et al. 2017), saddle between Zeppelinfjellet and Lundryggen (Breuss 2017).

*Porpidia flavicunda (Ach.) Gowan - 4, 6, 9, on siliceous stones. Widely distributed throughout Svalbard but it is not common.

Porpidia melinodes (Körb.) Gowan \& Ahti - 1, 6, on siliceous stones. Widely distributed throughout Svalbard and common.

Protopannaria pezizoides (Weber) P. M. Jørg. \& S. Ekman - 5 (KPABG 13515), 6 (KPABG 13569), 26 (KPABG 13545), on soil and mosses. Widely distributed throughout Svalbard and common.

Pseudephebe minuscula (Nyl. ex Arnold) Brodo \& D. Hawksw. - 1, 3 (KPABG 13508), 4 (KPABG 13617, 13620, 13621), 6 (KPABG 13567), 11, 14, 20 (KPABG 13632), 21, on siliceous stones. Widely distributed throughout Svalbard and common.

Pseudephebe pubescens (L.) M. Choisy - 1 (KPABG 13641), 4, 6, 15 (KPABG 13585), 16, 17 (KPABG 13501), 26, on siliceous stones. Widely distributed throughout Svalbard and common.

Rhexophiale rhexoblephara (Nyl.) Hellb. - 4 (KPABG 13513), on soil among mosses. Known from a few scattered sites of Svalbard: Sjuøyane (Elvebakk and Hertel 1996); Nordenskiöld Land - Grønfjordfjellet (Vězda 1967, Urbanavichene and Koroleva 2008); Oskar II Land - Ny-Ålesund (Øvstedal et al. 2009); Nordaustlandet - Damflya (Konoreva et al. 2019); Barentsøya - Frankenhalvøya (O-L138242, O-L138338); Kongsfjorden - ved Gåselva (BG-L69811) [1].

Rhizocarpon badioatrum (Flörke ex Spreng.) Th. Fr. - 1, 4, on siliceous stones. Widely distributed throughout Svalbard and common.

Rhizocarpon copelandii (Korb.) Th. Fr. - 6, 15 (KPABG 13583), 17 (KPABG 13630), 27 (KPABG 13527, 13529), on siliceous stones. Widely distributed throughout Svalbard and common.

Rhizocarpon eupetraeoides (Nyl.) Blomb. \& Forssell - 15 (KPABG 13581), on siliceous stone. Rare on Svalbard. Reported to the third time from Svalbard. Previously reported from Amsterdamøya (Hertel and Ullrich 1976); Nordaustlandet - Damflya (Konoreva et al. 2019).

Rhizocarpon ferax H. Magn. -4, 11, on siliceous stones. Widely distributed throughout Svalbard and not common. 
Rhizocarpon geminatum Körb. - 4 (KPABG 13620), 16 (KPABG 13558), on siliceous stones. Widely distributed throughout Svalbard.

Rhizocarpon geographicum (L.) DC. - 4 (KPABG 13521, 13617, 13620), 9 (KPABG 13552), 13 (KPABG 13642), 15 (KPABG 13584), 16 (KPABG 13559, 13561, 13563), 17 (KPABG 13503, 13630), 24 (KPABG 13506), 27 (KPABG 13530), on siliceous stones. Widely distributed throughout Svalbard.

Rhizocarpon grande (Flörke) Arnold - 11, on siliceous stone. Widely distributed throughout Svalbard.

*Rhizocarpon hochstetteri (Körb.) Vain. -4, on siliceous stone. Sporadically distributed throughout Svalbard.

Rhizocarpon inarense (Vain.) Vain. - 1, 4 (KPABG 13522), 6, 8, 10, 15 (KPABG 13582, 13584), 17 (KPABG 13457), on siliceous stones. Widely distributed throughout Svalbard.

Rhizocarpon intermediellum Räsänen - 16, on siliceous stone. Sporadically distributed throughout Svalbard.

* Rhizocarpon rittokense (Hellb.) Th. Fr. - 4 (KPABG 13618), 6, 16 (KPABG 13561), on siliceous stones. Known from a few scattered sites of Svalbard: Hornsund (Fries 1867); Sveagruva (Eurola 1971); Bockfjorden (Schuhwerk 1992); Nordenskiöld Land - Bolterdalen (Breuss 2017); Bünsow Land - Gipshuken (TROM-L564677); NyFriesland - Austbotnhytta (BG-L81673) [1].

Rhizocarpon superficiale (Schaer.) Vain. - 15, on siliceous stone. Sporadically distributed throughout Svalbard.

*Rhizocarpon viridiatrum (Wulfen) Körb. - 1, on siliceous stone. Rare on Svalbard. Reported to the second time from Svalbard. Previously reported from Nordenskiöld Land - Colesbukta (Konoreva and Chesnokov 2018).

Rhizoplaca melanophthalma (DC.) Leuckert \& Poelt - 12 (KPABG 13629), on siliceous stone. Widely distributed throughout Svalbard.

* Rinodina terrestris Tomin. - 20 (KPABG 13507), on mosses. Rare on Svalbard. Reported to the third time from Svalbard. Previously reported from Danskøya Kobbefjorden (Øvstedal et al. 2009); Ny-Friesland - Austbotnhytta (BG-L94924) [1].

Rinodina turfacea (Wahlenb.) Korb. - 8 (KPABG 13550), on soil and mosses. Widely distributed throughout Svalbard.

Rusavskia elegans (Link) S. Y. Kondr. \& Kärnefelt - 5 (KPABG 13510), 10 (KPABG 13544), 16, 21, on siliceous stones. Widely distributed throughout Svalbard.

Rusavskia sorediata (Vain.) S. Y. Kondr. \& Kärnefelt - 6 (KPABG 13566), on siliceous stone. Widely distributed throughout Svalbard.

*Schaereria fuscocinerea (Nyl.) Clauzade \& Cl. Roux - 17, on stone. Known from a few scattered sites of Svalbard: Hornsund (Fries 1867); Edgeøya (Aptroot and Alstrup 1991); Isfjorden (Hertel 1991); Recherchefjorden (Øvstedal et al. 2009); Nordenskiöld Land - Todalen (O-L116824), Green Harbour (O-L126298); Albert I Land - Red Bay (O-L150190); Wedel Jarlsberg Land - Reinodden (O-L130255) [1].

Solorina bispora Nyl. - 9 (KPABG 13542), 16 (KPABG 13565), on calcareous soil. Widely distributed throughout Svalbard.

Solorina crocea (L.) Ach. - 1 (KPABG 13478, 13482, 13639), 4 (KPABG 13608), 6 (KPABG 13578), 9, 25 (KPABG 13636), on acidic soil. Widely distributed throughout Svalbard.

Solorina spongiosa (Ach.) Anzi - 16, on calcareous soil. Widely distributed throughout Svalbard. 
Sphaerophorus fragilis (L.) Pers. - 4 (KPABG 13524), 8, 23 (KPABG 13634), on soil among mosses. Widely distributed throughout Svalbard.

Sphaerophorus globosus (Huds.) Vain. - 6 (KPABG 13572), 8 (KPABG 13550), on soil among mosses. Widely distributed throughout Svalbard.

Sporastatia polyspora (Nyl.) Grummann - 1, on siliceous stone. Widely distributed throughout Svalbard.

Sporastatia testudinea (Ach.) A. Massal. - 16, 25 (KPABG 13487), on siliceous stone. Widely distributed throughout Svalbard.

Stereocaulon alpinum Laurer - 25, on soil among mosses. Widely distributed throughout Svalbard.

Stereocaulon botryosum Ach. $\mathbf{- 4}$, on soil on boulder. Widely distributed throughout Svalbard.

* Stereocaulon cumulatum (Sommerf.) Timdal - 23 (KPABG 13500), on mosses. Rare on Svalbard. Reported to the third time from Svalbard. Previously reported from Bünsow Land - Gipsvika; Brøggerhalvøya - Ny-Ålesund (Øvstedal et al. 2009).

Stereocaulon glareosum (Savicz) H. Magn. - 1, 4, 6, 9, 12, 16, 20, on soil among mosses. Widely distributed throughout Svalbard.

Stereocaulon rivulorum H. Magn. - 14, on soil among mosses. Widely distributed throughout Svalbard.

Tetramelas insignis (Nägeli ex Hepp) Kalb - 1 (KPABG 13469, 13613), 5 (KPABG 13514), on soil and mosses. Widely distributed throughout Svalbard.

Tetramelas papillatus (Sommerf.) Kalb - 1, on soil. Widely distributed throughout Svalbard.

Thamnolia vermicularis (Sw.) Schaer. - 4 (KPABG 13623), 6 (KPABG 13573), 21 (KPABG 13553), 23 (KPABG 13499), 26 (KPABG 13536), on soil, among mosses. Widely distributed throughout Svalbard.

* Toninia squalida (Ach.) Massal. - 4, on calcareous soil. Rare on Svalbard. Reported to the third time from Svalbard. Previously reported from Prins Karls Forland - Carls Forland (Timdal 1991); Dickson Land - Tysneset (TROM-L565889) [1].

Tremolecia atrata (Ach.) Hertel - 4 (KPABG 13521), 9 (KPABG 13552), 10, 14, 24 (KPABG 13506), on siliceous stones. Widely distributed throughout Svalbard.

Umbilicaria arctica (Ach.) Nyl. - 25, on siliceous stone. Widely distributed throughout Svalbard.

Umbilicaria cylindrica (L.) Delise ex Duby - 1 (KPABG 13479), 4, 6 (KPABG 13577), 8 (KPABG 13511), 9, on siliceous stones. Widely distributed throughout Svalbard.

Umbilicaria decussata (Vill.) Frey - 4, on siliceous stone. Widely distributed throughout Svalbard.

Umbilicaria hyperborea (Ach.) Hoffm - 1, 4, 23, on siliceous stones. Widely distributed throughout Svalbard.

Umbilicaria lyngei Scholander - 6, 23, on siliceous stones. Widely distributed throughout Svalbard.

Umbilicaria proboscidea (L.) Schrad. - 4, 6, 15, 17, on siliceous stones. Widely distributed throughout Svalbard.

Umbilicaria torrefacta (Lightf) Schrad. - 6 (KPABG 13579), on siliceous stone. Widely distributed throughout Svalbard.

Umbilicaria virginis Schaerer $\mathbf{- 8 , 2 1}$, on siliceous stones. Widely distributed throughout Svalbard.

*Verrucaria hydrela Ach. - 18, on wet siliceous stone. Rare on Svalbard. Reported to the third time from Svalbard. Previously reported from Ny-Friesland $-\mathrm{S}$ of the mouth 
of Ringhorndalen (Øvstedal et al. 2009); Nordenskiöld Land - Kolfjellet (OL138803) [1].

*Verrucaria nigrescens Pers. - 16, on sandstone. Rare on Svalbard. Known from a few scattered sites of Svalbard: Bjørnøya (Summerhayes and Elton 1923, Lynge 1926); Nathorst Land - Mitterhuken (Øvstedal et al. 2009), Forsbladhamna (O-L128320) [1]; Nordenskiöld Land - Berzeliusfjellet (Øvstedal et al. 2009); Wedel Jarlsberg Land Calypso - Sørhuken (O-L125325) [1].

Xanthomendoza borealis (R. Sant. \& Poelt ) Søchting et al. - 18 (KPABG 13541), on siliceous stone. Known from a few scattered sites of Svalbard: Amsterdamøya; Nordenskiöld Land - Longyearbyen (Elvebakk and Hertel 1996); Nordaustlandet Damflya (Konoreva et al. 2019); Oskar II Land - Zeppelinfjellet (Breuss 2017); Indre Norskøya - Sabineodden (Øvstedal et al. 2009); Bünsow Land - Gipshuksletta (TROM-L564667); Andree Land - Munningen av Kartdalen (O-L127847) [1].

\section{Conclusion}

As a result of our research, we found 2 species new to Svalbard and 27 species that are rare and extremely rare on Svalbard. Among them the new species for Svalbard Alectoria gowardii which was described by Halonen et al. (2009) as Gowardia arctica Halonen et al. It was probably previously determined as Alectoria nigricans or other closely related taxa (see comment under species). The second species new to Svalbard Peltigera elisabethae, is probably more widespread, but often overlooked. From the 27 rare and extremely rare species, 15 are saxicolous lichens (for example, Aspicilia cinerea, Henrica melaspora, Lecidea ecrustacea, Lendemeriella exsecuta, Miriquidica deusta, etc). They are small in size and difficult for collection and identification. Species such as Cetraria nigricans, Cladonia arbuscula, Cystocoleus ebeneus, Peltigera frippii, Peltigera kristinssonii, and Peltigera lyngei, prefer humid habitats as well as those protected from the wind. They were found by us near Ringgåsvatnet Lake, where there are suitable conditions. Toninia squalida was also found on the calcareous soil on the rocks near Ringgåsvatnet. Thus, in this study area, the Ringgåsvatnet Lake is very interesting for lichenologists because of the suitable conditions (the presence of calcareous rocky outcrops, humidity and protection from the wind). Another extremely rare species on the Svalbard is Gyalecta erythrozona, that was found on rocks near the sea shore.

\section{References}

Aнті, T. (1961): Taxonomic studies on reindeer lichens (Cladonia, subgenus Cladina). Annales Botanici Societatis Zoologicae-Botanicae Fennicae Vanamo, 32(1): 1-160.

AleXandrova, V. D. (1977): Geobotanical zoning of the Arctic and Antarctic. Nauka, Leningrad. $189 \mathrm{p}$.

Aptroot, A., Alstrup, V. (1991): Lichens from Edgeøya, Svalbard. Graphis Scripta, 3: 73-75.

BREuss, O. (2017): Flechten aus Spitzbergen im Herbarium des Oberösterreichischen landesmuseums (LI), gesammelt 1975 von Werner Repetzky. Stapfia, 107: 163-168.

Czernyadjeva, I. V., Ahti, T., Boldina, O. N., Chesnokov, S. V., Davydov, E. A., Doroshina, G. Ya., Fedosov, V. E., Khetagurov, Kh. M., Konoreva, L. A., Kotkova, V. M., Kuzmina, E. Yu., Lavrentiev, M. V., Liksakova, N. S., Nikolayev, I. A., Popova, N. N., Safronova, T. V., Shadrina, S. N. and Yakovchenko, L. S. (2020): New cryptogamic records. 6. Novosti sistematiki nizshikh rastenii, 54(2): 537-557. 
Dallmann, W. K., Ohta, Y., Elvevold, S. and Blomeier, D. (2002): Bedrock of Svalbard and Jan Mayen 1: 750 000. - Norsk Polarinstitutt Temakart N33

ElvebakK, A., Hertel, H. (1996): A catalogue of Svalbard plants, fungi, algae and cyanobacteria. Part 6. Lichens. Norsk Polarinstitutt Skrifter, 198: 271-359.

ENGELSKJøn, T. (1986): Eco-geographical relations of the Bjørnøya vascular flora, Svalbard. Polar Research, 5: 79-127.

EuRola, S. (1971): Die Vegctation einer Sturzhalde (Sveagruva, Spitzbergen, 7753' n. Br.). Aquilo. Seria Botanica, 10: 8-28.

Fries, T. M. (1867): Lichenes Spitsbergenses. Kongl Svenska Vetenskaps-Akademiens Handlingar, 72: $1-53$.

GYelNiK, V. (1932): Enumeratiolichenum europaeorum novorum rariorumque. 1. Annales Mycologici, 30: 442-459.

HAFELLNER, J. (1982): Flechtenfunde am Bockfjord, Spitzbergen. Ergebnisse der österreichischen Spitzbergen-Expedition 1979, I. Phyton, 22: 23-50.

HAGEN, A. (1950): Notes on arctic fungi. Norsk Polarinstitutt Skrifter, 93: 1-23.

Halonen, P., Myllys, L., Velmala, S. and Hyvärinen, H. (2009): Gowardia (Parmeliaceae) - a new alectorioid lichen genus with two species. The Bryologist, 112(1): 138-146.

HARTMANN, H. (1980): Beitrag zu Kenntnis der Pftanzengesellschaften Spitlbergens. Phylocoenologia, 8: 65-147.

Hertel, H. (1970): Beitrage zur Kenntnis der Flechtenfamilie Lecideaceae III. Herzogia, 2: 37-62.

Hertel, H. (1977): Bemerkenswerte Flechtenfunde aus dem Gebiet des Kongsfjordes und des Isfjordes (Spitzbergen). Herzogia, 4: 367-401.

HeRTEL, H. (1991): Lecidea in der Arktis III (lecideoide Flechten, Lecanorales). Mitteilungen (aus) der Botanischen Staatssammlung München, 30: 297-333.

Hertel, H. and Ulbrich, H. (1976): Flechten von Amsterdamøya (Svalbard). Mitteilungen aus der Botanischen Staatssammlung München, 12: 417-512.

Inoue, T., Uchida, M., Inoue, M., KaneKo, R., Kudoh, S., Minami, Y. and Kanda, H. (2019): Vegetation data of high Arctic lichens on Austre Brøggerbreen glacier foreland, Ny-Ålesund, Svalbard, in 1994. Polar Data Journal, 3: 1-11.

Kondratyuk, S. Y., LöKÖs, L., FArKas, E., Kärnefelt, I., Thell, A., Yamamoto, Y. and Hur, J.-S. (2020): Three new genera of the Teloschistaceae proved by three gene phylogeny. Acta Botanica Hungarica, 62(1-2): 109-136.

Konoreva, L. A. (2011): A contribution to the lichens of vicinity of the former Pyramiden settlement (Spitsbergen Island, Svalbard Archipelago). Novosti sistematiki nizshikh rastenii, 45: 183-193.

Konoreva, L. A., Chesnokov, S. V. (2018): Lichens of vicinity of the former township Kolsbey (West Spitsbergen). Bulletin of the Kola Scientific Center of the Russian Academy of Sciences, 3(10): 31-42.

Konoreva, L., Kozhin, M., Chesnokov, S. and Hong, S. G. (2019): Lichens and vascular plants in Duvefjorden area on Nordaustlandet, Svalbard. Czech Polar Reports, 9(2): 182-199.

KRZEWICKA, B. and MACIEJOWSKI, W. (2008): Lichen species from the northeastern shore of Sørkapp Land (Svalbard). Polar Biology, 31: 1319-1324.

KuKWA, M., JABŁOŃSKA, A. (2008): New or interesting records of lichenicolous fungi from Poland VI. Herzogia, 21: 167-179.

Lumbsch, H. T. and Huhndorf, S. M. (2010): Myconet Volume 14. Part One. Outline of Ascomycota-2009. Part Two. Notes on Ascomycete Systematics. Nos. 4751-5113. Fieldiana Life and Earth Sciences, 1: 1-64.

Lynge, B. (1924): Lichens from Spitsbergen I. Result. Norske Slalsundersl. Spitsbergeneksped, 1(5): $1-21$.

LyNGE, B. (1926): Lichens from Bear Island (Bjørnøya) collected by Norwegian and Swedish expeditions, chiefly by Th. M. Fries during the Swedish polar expedition of 1868 . Resultater av de Norske Statsunderstøttede Spitsbergen Ekspeditioner, 1(9): 1-78. 
LYNGE, B. (1938): Lichens from the west and north coasts of Spitsbergen and the North-East Land collected by numerous expeditions. 1. The macrolichens. Skrifter Norsk videnskaps-akademi $i$ Oslo. 1. Mathematisk-naturvidenskabelig klasse, 6: 1-136.

LyNGE, B. (1939): A small contribution to the lichen flora of the eastern Svalbard islands. Meddelelse Norges Svalbard-Og Ishavs-Undersøkelser, 44: 1-12.

Maciejowski, W., Osyczka, P., Smykla, J., Ziaja, W., Ostafin, K. and Krzewicka B. (2018): Diversity and distribution of lichens in recently deglaciated areas of southeastern Spitsbergen. Acta Societatis Botanicorum Poloniae, 87: 3596.

MaKarova, I. I., Himelbrant, D. E. (2008): Stereocaulaceae Chevall. In: N. S. Golubkova (ed.): Handbook of the lichens of Russia. Vol. 10. Agyriaceae, Anamylopsoraceae, Aphanopsidaceae, Arthrorнaphidaceae, Brigantiaeaceae, Chrysotrichaceae, Clavariaceae, EctolecHiaceae, Gompнillaceae, Gypsoplacaceae, Lecanoraceae, Lecideaceae, Mycoblastaceae, Phlycridaceae, Physciaceae, Pilocarpaceae, Psoraceae, Ramalinaceae, Stereocaulaceae, V Ezdaeaceae, Tricholomataceae. Nauka, St. Petersburg; pp. 443-463.

Matveyeva, N. V., Zanokha, L. L., Afonina, O. M., Potemkin, A. D., Patova, E. N., Davydov, D. A., Andreeva, V. M., Zhurbenko, M. P., Konoreva, L. A., Zmitrovich, I. V., Ezhov, O. N., Shiryaev, A. G. and Kirtsideli, I. Yu. (2015): Plants and fungi of the polar deserts in the northern hemisphere. Publishing "Maraphon", Saint-Petersburg. 320 p.

Nordin, A. (2010): Lichens new to Svalbard and Norway. Graphis Scripta, 24: 28-31.

NowAK, J. (1965): The lichens from Hornsund (S.W.-Spitsbergen) collected during the Polish polar expeditions in 1957 and 1958. Fragmenta Floristica et Geobotanica, 11: 171-190.

Olech, M. 1990: Lichens of the NW Sørkapp Land (Spitsbergen). Zeszyty Naukowe UJ, Prace Botaniczne, 21: 197-210.

OsyczKA, P. (2006): The lichen genus Cladonia (Cladoniaceae, lichenized Ascomycota) from Spitsbergen. Polish Polar Research, 27(3): 207-242.

Øvstedal, D. O., Tønsberg, T. and Elvebakk, A. (2009): The lichen flora of Svalbard. Sommerfeltia, 33: 1-393.

Redchenko, O., KoŠnar, J. and Gloser, J. (2010): A contribution to lichen biota of the central part of Spitsbergen, Svalbard Archipelago. Polish Polar Research, 31(2): 159-168.

SAAG, L., SAAG, A. and RANDlane, T. (2009): World survey of the genus Lepraria (Stereocaulaceae, lichenized Ascomycota). The Lichenologist, 41(1): 25-60

SANTESSON, R. (1939): Amphibious pyrenolichens I. Arkiv för Botanik, 29A(10): 1-67.

SCHOLANDER, P. F. (1934): Vascular plants from northem Svalbard with remarks on the vegetation in North-East Land. Skrifter om Svalbard og Ishavet, 62: 1-153.

SChuhwerk, F. (1992): Die Berucksichtigung der Okologie in der Lichenometrie: Datierung mit Sukzessionsstadien von F1echtengesellschaften. In: W. D. Blümel (ed.): Geowissenschaftliche Spitzbergen-Expedition 1990 und 1991 "Stofftransporte Land -Meer in polaren Geosystemen". Zwischenbericht. Stuttg. Geogr. Studien 117. pp. 161-175.

Sérusiaux, E., Diederich, P., Brand, A. M. and van den Boom, P. P. G. (1999): New or interesting lichens and lichenicolous fungi from Belgium and Luxembourg VIII. Lejeunia, 162: $1-95$.

Singh, S. M. and RavindRA, R. (2013): Chapter 7. Impact of Climate Change on Lichen and Moss Communities in Ny-Ålesund, Arctic: Some Preliminary Observations. In: R. Sinha and R. Ravindra (eds.): Earth System Processes and Disaster Management. Society of Earth Scientists Series 1, Springer-Verlag Berlin Heidelberg, pp. 93-100.

Smith, C. W., Aptroot, A., Coppins, B. J., Fletcher, A., Gilbert, O. L., James, P. W. and Wolseley, P.A. (eds.) (2009): The lichens of Great Britain and Ireland. The British Lichen Society, London, $1046 \mathrm{p}$.

Søchting, U. (1989): Lignicolous species of the lichen genus Caloplaca from Svalbard. Opera Botanica, 100: 241-257.

Søchting, U. (1992): On the identity and distribution of some Nordic Caloplaca species. Graphis Scripta, 4: 91-92.

Søchting, U., Balschmidt Lorentsen, L. and Arup, U. (2008): The lichen genus Caloplaca (Ascomycota, Lecanoromycetes) on Svalbard. Notes and additions. Nova Hedwigia, 87: 69-96. 
Summerhayes, V. S., Elton, C. S. (1923): Contributions to the ecology of Spitsbergen and Bear Island. Journal of Ecology, 11:214-286.

Timdal, E. (1991): A monograph of the genus Toninia (Lecideaceae, Ascomycetes). Opera Botanica, 110: 1-137.

Urbanavichene, I. N. and Koroleva, N. E. (2008): Lichens. In: N. E. Koroleva, N. A. Konstantinova, O. A. Belkina, D. A. Davydov, A. Yu. Likhachev, A. N. Savchenko, I. N. Urbanavichene (eds.): Flora of Vegetation of Grønfjord area (Spitsbergen archipelago). K\&M, Apatity, pp. 81-92.

VĚZDA, J. (1967): Flechtensystematischc Studien VI. Die GaUung Sagiolechia Massal. Folia Geobotanica et Phytotaxonomica, 2: 383-396.

Vitikainen, O. (1994): Taxonomic revision of Peltigera (lichenized Ascomycotina) in Europe. Annales Botanici Fennici, 152: 1-96.

Vitikainen, O. (2007): Peltigeraceae. In: T. Ahti, P. M. Jørgensen, H. Kristinsson, R. Moberg, U. Søchting and G. Thor (eds.): Nordic Lichen Flora 3. Cyanolichens. The Nordic Lichen Society, Museum of Evolution, Uppsala University, Uddevalla, pp. 113-131.

Węgrzyn, M., WietrzyK, P., AdAmska, E. and Nicia, P. (2015): New records of driftwood lichens in the Kaffiøyra Plain (NW Spitsbergen, Svalbard). Polish Polar Research, 36(2): 189195.

Westberg, M. (2007): Candelariella (Candelariaceae) in western United States and northern Mexico: The polysporous species. The Bryologist, 110(3): 375-390.

Westberg, M., Ahti, T. and Thell, A. (2011): Hypogymnia. In: A. Thell and R. Moberg (eds): Nordic Lichen Flora 4. Parmeliaceae. The Nordic Lichen Society, Museum of Evolution, Uppsala University, pp. 56-62.

Westberg, M., Moberg, R., Myrdal, M., Nordin, A. and Ekman, S. (2021): Santesson's Checklist of Fennoscandian Lichen-Forming and Lichenicolous Fungi. Uppsala University, Museum of Evolution. 933 p.

WiETRZYK, P., WĘGRZYN, M. and LisowSKA, M. (2017): Lichen diversity on glacier moraines in Svalbard. Cryptogamie, Mycologie, 38(1): 67-80.

Zhurbenko, M. P., Brackel, W. v. (2013): Checklist of Lichenicolous Fungi and Lichenicolous Lichens of Svalbard, Including New Species, New Records and Revisions. Herzogia, 26(2): 323-359.

Ziaja, W., Maciejowski, W. and Ostafin, K. (2009): Coastal Landscape Dynamics in NE Sørkapp Land (SE Spitsbergen), 1900-2005. A Journal of the Human Environment, 38(4): 201208.

\section{Web sources / Other sources}

[1] 'Svalbard Lichen Database' (SLD, http://www.nhm2.uio.no/botanisk/nxd/sval_L/sld_e.htm)

[2] 'TopoSvalbard' (https://toposvalbard.npolar.no/) 